\title{
Editorial
}

\section{Super-Resolution Enhancement of Digital Video}

\author{
Russell C. Hardie, ${ }^{1}$ Richard R. Schultz, ${ }^{2}$ and Kenneth E. Barner ${ }^{3}$ \\ ${ }^{1}$ Department of Electrical and Computer Engineering, University of Dayton, 300 College Park, Dayton, \\ OH 45469-0026, USA \\ ${ }^{2}$ Department of Electrical Engineering, University of North Dakota, Upson II Room 160, P.O. Box 7165, \\ Grand Forks, ND 58202-7165, USA \\ ${ }^{3}$ Department of Electrical and Computer Engineering, University of Delaware, 140 Evans Hall, Newark, \\ DE 19716-3130, USA
}

Received 10 June 2007; Accepted 10 June 2007

Copyright ( 2007 Russell C. Hardie et al. This is an open access article distributed under the Creative Commons Attribution License, which permits unrestricted use, distribution, and reproduction in any medium, provided the original work is properly cited.

When designing a system for image acquisition, there is generally a desire for high spatial resolution and a wide field of view. To satisfy these competing requirements, a camera system typically must employ small $f$-number optics, which produces an image with very high spatial-frequency bandwidth at the focal plane. To avoid aliasing caused by undersampling, the corresponding focal plane array (FPA) must be sufficiently dense. However, cost and fabrication complexities may make this impractical. More fundamentally, smaller detectors capture fewer photons, which can lead to potentially severe noise levels in the acquired imagery. Considering these factors, one may choose to accept a certain level of undersampling or to sacrifice some optical resolution and/or field of view.

In super-resolution (SR) image reconstruction, postprocessing is used to obtain images with resolutions that go beyond the conventional limits of the uncompensated imaging system. In some systems, the primary limiting factor is the optical resolution of the image in the focal plane as defined by the cut-off frequency of the optics. We use the term "optical SR" to refer to SR methods that aim to create an image with valid spatial-frequency content that goes beyond this cut-off frequency. Such techniques typically must rely on extensive a priori information. In other image acquisition systems, the limiting factor may be the density of the FPA, subsequent postprocessing requirements, or transmission bit rate constraints that require data compression. We refer to the process of overcoming the limitations of the FPA in order to obtain the full resolution afforded by the selected optics as "detector SR." Some methods may seek to perform both optical and detector SR.
Detector SR algorithms generally process a set of lowresolution aliased frames from a video sequence to produce a high-resolution frame. When subpixel relative motion is present between the objects in the scene and the detector array, a unique set of scene samples is acquired for each frame. This provides the mechanism for effectively increasing the spatial sampling rate of the imaging system without reducing the physical size of the detectors.

With the proliferation of digital imaging and video, SR has become a rapidly growing field. Recent advances in SR include innovative algorithms, generalized methods, real-time implementations, and novel applications. The purpose of this special issue is to present leading research and development in the area of super-resolution image reconstruction as applied to digital video. Specific topics addressed in this special issue include image registration, regularization, photometric diversity, detector nonuniformity, compression, optical design, and performance metrics.

Two of the papers in this special issue address topics related to subpixel image registration for SR. In particular, the paper by L. C. Pickup et al. presents a joint registration and SR approach in a Bayesian framework, as well as an alternative approach using Bayesian marginalization over unknown registration parameters. The paper by M. Das Gupta et al. presents a supervised learning based approach to subpixel motion estimation for video super-resolution. Two other papers in this issue explore various cost functions and regularization methods for SR. V. Patanavijit and S. Jitapunkul use a Lorentzian error norm for measuring the difference between the projected estimate of the high-resolution image and each low-resolution image. They also employ both Tikhonov and Lorentzian-Tikhonov regularization to 
remove artifacts from the SR estimate and improve the convergence rate of the corresponding iterative algorithms. The paper by $\mathrm{M}$. $\mathrm{Ng}$ et al. employs total variation regularization in the reconstruction model, as a variant on the more traditional regularized least-squares-based techniques.

Images used for SR may be affected by factors other than the traditional aliasing, blur, and noise that must be addressed. For example, multiple images may be captured under photometric variation. This may include changing external illumination conditions and/or camera calibration differences. M. Gevrekci and B. K. Gunturk propose a novel nonlinear technique for addressing these variations. Another problem often encountered, particularly for infrared imagers, is detector nonuniformity. R. C. Hardie and D. R. Droege present a Bayesian approach for simultaneously estimating detector nonuniformity parameters and SR imagery.

SR with image compression is another important area addressed in this issue. L. Shao presents an adaptive resolution up-conversion algorithm that is robust to compression artifacts. The method is based on classification of local image patterns and uses a least-mean-square training procedure. The paper by N. Maor et al. considers compression at the source for digital camcorders. Their system is based on a variable spatiotemporal sampling strategy, which combines infrequent high-resolution frames with more frequent lowresolution frames.

As SR techniques become more prevalent, optical designs are starting to be influenced by the potential for SR postprocessing. The paper by N. A. Ahuja and N. K. Bose explores the design of an optical system with a large field of view using a lenslet array, applying multiframe SR techniques to the resulting images. The proposed system is capable of realizing both a specified resolution and specified field of view. Finally, the paper by A. van Eekeren et al. explores performance metrics for SR on real-world data. Their proposed metric is based on an observer task referred to as triangle orientation discrimination. This provides a useful means for quantitatively comparing the performance of SR reconstruction methods under varying conditions.

We sincerely hope that you enjoy and learn from this collection of SR papers. SR from digital video is a relatively new field, in only its third decade of existence. There is no doubt that as imaging sensor technologies, optical fabrication techniques, and computational algorithms mature, SR will find its way into digital video products such as cameras and digital cable set-top boxes. These papers on the fundamental SR topics of image registration, regularization, photometric diversity, detector nonuniformity, compression, optical design, and performance metrics serve as pioneers in the dynamic and evolving field of SR image reconstruction research and development. We are proud to present them to the image and video processing research community.

Russell C. Hardie Richard R. Schultz Kenneth E. Barner
Russell C. Hardie graduated magna cum laude from Loyola College in Baltimore Maryland in 1988 with a B.S. degree in engineering science. He obtained his M.S. and Ph.D. degrees in electrical engineering from the University of Delaware in 1990 and 1992, respectively. Dr. Hardie served as a Senior Scientist at Earth Satellite Corporation in Maryland prior to his appointment at the University of Dayton in 1993.

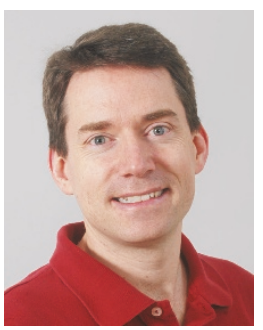

He is currently a Full Professor in the Department of Electrical and Computer Engineering and holds a joint appointment with the Electro-Optics Program. Along with several collaborators, Dr. Hardie received the Rudolf Kingslake Medal and Prize from SPIE in 1998 for work on multiframe image resolution enhancement algorithms. Dr. Hardie recently received the University of Dayton's Top University-Wide Teaching Award, the 2006 Alumni Award in Teaching. In 1999, he received the School of Engineering Award of Excellence in Teaching at the University of Dayton and was the recipient of the first Professor of the Year Award in 2002 from the student chapter of the IEEE at the University of Dayton. His research interests include a wide variety of topics in the area of digital signal and image processing. His research work has focused on image enhancement and restoration, pattern recognition, and medical image processing.

Richard R. Schultz serves as an Associate Professor and Chair of electrical engineering at the University of North Dakota (UND) in Grand Forks. Dr. Schultz received the B.S.E.E. degree from UND in 1990 and the M.S.E.E. and Ph.D. degrees from the University of Notre Dame in 1992 and 1995, respectively. He joined the UND faculty in 1995, and his teaching and research interests are in aerospace payload and sensor de-

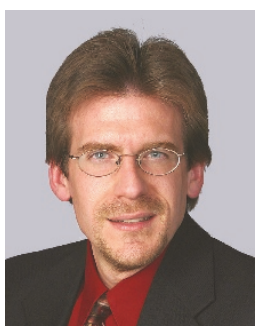

velopment, control systems, digital image and video processing, embedded systems design, student-generated intellectual property, systems engineering, and technology entrepreneurship. Prior to his appointment as Department Chair, Dr. Schultz served as a Coproject Director of the North Dakota Experimental Program to Stimulate Competitive Research (EPSCoR), from January 2004 through August 2005. In this role, he had the unique opportunity of fostering interdisciplinary campus and statewide research, development, and commercialization partnerships. Dr. Schultz is currently involved in the Unmanned Aircraft Systems Center of Excellence at the University of North Dakota, an interdisciplinary research center that includes the John D. Odegard School of Aerospace Sciences, the School of Engineering and Mines, the College of Nursing, and the Department of Psychology. Within this center, Dr. Schultz helps supervise the design of unmanned aerial vehicle payloads and sensors, conducts research on the enhancement of surveillance digital video for automatic target recognition and tracking, and guides the development of sense and avoid technologies for unmanned aircraft.

Kenneth E. Barner received his B.S.E.E. degree from Lehigh University, Bethlehem, $\mathrm{Pa}$, USA in 1987. He received the M.S.E.E. and Ph.D. degrees from the University of Delaware, Newark, Delaware, in 1989 and 1992, respectively. $\mathrm{He}$ is currently a Professor in the Department of Electrical and Computer Engineering at the University of Delaware. He was the

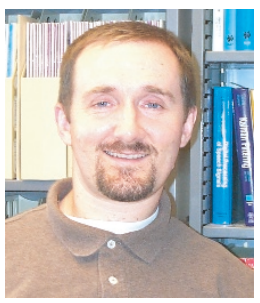


cochair of the 2001 IEEE-EURASIP Nonlinear Signal and Image Processing (NSIP) Workshop and a Guest Editor for a special issue of the EURASIP Journal of Applied Signal Processing on Nonlinear Signal and Image Processing. He is a Member of the Nonlinear Signal and Image Processing Board and is coeditor of the book Nonlinear Signal and Image Processing: Theory, Methods, and Applications, CRC Press, 2004. He was the Technical Program cochair for ICASSP 2005 and is currently serving on the IEEE Signal Processing Theory and Methods (SPTM) and IEEE Bio-Imaging and Signal Processing (BISP) technical committees. Dr. Barner has served as an Associate Editor of the IEEE Transactions on Signal Processing, the IEEE Transaction on Neural Systems and Rehabilitation Engineering, and the IEEE Signal Processing Magazine. He is currently the Editor-in-Chief of the journal Advances in HumanComputer Interaction, a Member of the Editorial Board of the EURASIP Journal of Applied Signal Processing, and is serving as a Guest Editor for that journal on the Empirical Mode Decomposition and the Hilbert-Huang Transform special issue. His research interests include signal and image processing, robust signal processing, nonlinear systems, communications, human-computer interaction, haptic and tactile methods, and universal access. 